\title{
Inverse relationship between serum high density lipoprotein and negative syndrome in antipsychotic-naive schizophrenia
}

\author{
Ganesan Venkatasubramanian*, Rashmi Arasappa, \\ Naren P. Rao, Rishikesh V. Behere, Sunil Kalmady \\ and Bangalore N. Gangadhar
}

The Metabolic Clinic in Psychiatry, Department of Psychiatry, National Institute of Mental Health and Neurosciences, Bangalore, Karnataka, India

\begin{abstract}
Background: Recent literature suggests a role for apolipoprotein $\mathrm{L}$ (apoL) aberrations in the pathogenesis of schizophrenia. ApoL is almost exclusively associated with apolipoprotein A-I in high-density lipoproteins (HDLs). The objective of this study was to examine the correlation between symptom scores and serum HDL in antipsychoticnaive schizophrenia patients.

Methods: In this cross-sectional study, 60 antipsychoticnaive schizophrenia patients were systematically examined for their symptom scores, with good inter-rater reliability. Concurrently, an overnight fasting serum lipid profile from these patients was assessed.

Results: Serum HDL had a significant inverse correlation with a total negative syndrome score $(\rho=-0.43 ; \mathrm{p}=0.001)$. Conclusions: The study observation supports the potential role for HDL abnormalities in the genesis of negative symptoms in schizophrenia.

Clin Chem Lab Med 2010;48:95-8.
\end{abstract}

Keywords: apolipoprotein L; high-density lipoprotein; negative syndrome; schizophrenia.

\section{Introduction}

Lipid abnormalities have been hypothesized to play a central role in the pathogenesis of schizophrenia (1-3). Apolipoprotein $\mathrm{L}$ (apoL) is a $42-\mathrm{kDa}$ plasma apolipoprotein that has four forms of apoL, namely apoL I-IV, which are encoded by separate genes on chromosome 22 (4). ApoL-I is expressed ubiquitously in the central nervous system, and robust up-regulation of the APOL-I gene has been demon-

*Corresponding author: Dr. Ganesan Venkatasubramanian, MD, The Metabolic Clinic in Psychiatry, Department of Psychiatry, National Institute of Mental Health and Neurosciences, Bangalore - 560029, Karnataka, India

Phone: +91 80 26995256, Fax: +91 8026564830 ,

E-mail: venkat.nimhans@yahoo.com

Received July 14, 2009; accepted September 16, 2009;

previously published online November 25, 2009 strated in the prefrontal cortex of patients with schizophrenia (5).

ApoL is almost exclusively associated with apoA-I in high-density lipoproteins (HDLs) (6). Interestingly, HDLs have been demonstrated to have an adaptive influence on certain brain structures such as the hippocampus (7). Indeed, various lines of evidence support the possibility of a signalling exchange between peripheral and brain lipids (7). Thus, assessment of serum lipids might potentially indicate the status of lipids in the brain lipid. Indeed, such relationships have been demonstrated in studies examining patients with dementia (8).

HDL is intricately associated with the prefrontal cortex, because of its link with apoL, and can adaptively influence the hippocampus. Also important, prefrontal (9) as well as hippocampal (10) abnormalities have consistently been demonstrated in patients with schizophrenia. In addition, these structural abnormalities of the brain have been linked to the positive and negative syndrome of schizophrenia (9-11). Since peripheral and brain lipids have signalling exchange between them (7), it is possible that serum HDL might show an association with symptom scores in patients with schizophrenia.

To the best of our knowledge, the symptom correlate of serum HDL in antipsychotic-naive schizophrenia patients has not been examined. The objective of this study was to evaluate an association between symptom scores and serum HDL in antipsychotic-naive patients with schizophrenia.

\section{Materials and methods}

Patients were recruited from individuals attending the clinical services of the National Institute of Mental Health and Neurosciences (India) and who fulfilled DSM-IV (Diagnostic and Statistical Manual of Mental Disorders) criteria for schizophrenia. Patients that were never treated with any psychotropic medication, including antipsychotics, and did not have substance abuse, were referred by the screening clinical psychiatrist for participation in the study. The patients were recruited over a period of 15 months, from September 2007 to November 2008. The diagnosis of schizophrenia was established by a qualified psychiatrist using Mini International Neuropsychiatric Interview Plus (12). The diagnosis was confirmed by another psychiatrist through an independent clinical interview. The details related to onset of illness and antipsychotic-naive status were ascertained with reliable information obtained from at least two adult relatives.

Psychosis symptoms were assessed using the Scale for Assessment of Positive Symptoms (SAPS) (13) and the Scale for Assessment of Negative Symptoms (SANS) (14). The SAPS total score 
was calculated by adding the following sub-scores: hallucinations, delusions, bizarre behavior, and formal thought disorder. The SANS total score was calculated by adding the following sub-scores: affective flattening, alogia, avolition-apathy, anhedonia-asociality and attention impairments. All the patients were rated by RA who had established a good inter-rater reliability (intra-class correlation coefficient $>0.9$ ) with another trained rater by examining, in turn, 10 patients.

For all subjects, weight $(\mathrm{kg})$ and height $(\mathrm{m})$ were measured and body mass index (BMI) $\left(\mathrm{kg} / \mathrm{m}^{2}\right)$ was calculated. The propensity to develop diabetes mellitus was assessed in all subjects using the Simplified Indian Diabetes Risk Score (SIDRS) (15). SIDRS is calculated based on the following parameters that are recommended by the American Diabetes Association: age, waist circumference, family history of diabetes mellitus and physical activity. SIDRS is a validated tool to assess the risk for developing diabetes mellitus in an Indian population. Though SIDRS is yet to be validated in people with mental illness, the simplicity of the questions as well as the scoring system enabled us to use it with ease in patients with schizophrenia (16). In addition, the information to calculate SIDRS was obtained from at least two adult relatives of the patient to ensure reliability. None of the subjects (patients and controls) had a family history of diabetes in any of their first-degree relatives. All subjects had a SIDRS of $\leq 50$.

None of the patients had alcohol abuse/dependence. None used stimulants or opiate drugs. None had history or clinical feature suggestive of a neurological/medical disorder. None had abnormal movements as assessed by Abnormal Involuntary Movements Scale (17). Clinical assessments and collection of blood samples were performed on the same day, before starting antipsychotics. Following a complete description of the study to the subjects, written informed consent was obtained. The Institute's Ethics Committee approved the study.

Blood samples were collected from all subjects between 0800 and $0900 \mathrm{~h}$ (A.M.) after $12 \mathrm{~h}$ overnight fast. Blood was collected from an ante-cubital vein into vacutainer tubes (Becton and Dickinson, Franklin Lakes, NJ, USA). After centrifugation of blood, serum was used for biochemical analyses. Glucose, liver and renal function tests, and serum lipid profiles were analyzed using the Olympus AU400 analyzer (Olympus Europa GmbH, Hamburg, Germany). Statistical analyses were performed using the Statistical Package for Social Sciences (version-11) (SPSS Inc, Chicago, IL, USA). Spearman's test and partial correlation analysis were used for correlation analyses. The statistical significance was set at $\mathrm{p}<0.05$ (two-tailed).

\section{Results}

The demographic characteristics of the patients were as follows: age (mean $\pm \mathrm{SD}$ ) $-29.9 \pm 7.4$ years; gender ratio (men:women) - 33:27. The Indian Diabetes Risk Score of the patients was $38.0 \pm 15.6$ and the BMI $\left(\mathrm{kg} / \mathrm{m}^{2}\right)$ was $18.6 \pm 3.5$. The psychopathology scores were as follows: SAPS total score - 28.4 \pm 15.1 ; SANS total score $65.5 \pm 28.8$. The fasting serum lipid profile was as follows: $\mathrm{HDL}-1.2 \pm 0.3 \mathrm{mmol} / \mathrm{L}$; total cholesterol - $4.2 \pm 1.1$ $\mathrm{mmol} / \mathrm{L}$; triglycerides $-1.1 \pm 0.6 \mathrm{mmol} / \mathrm{L}$; very low-density lipoprotein (VLDL) $-0.5 \pm 0.3 \mathrm{mmol} / \mathrm{L}$. There was significant negative correlation between SAPS and SANS total scores $(\rho=-0.64 ; \mathrm{p}<0.001)$. Serum HDL showed a significant inverse correlation with SANS total score $(\rho=-0.43$; $\mathrm{p}=0.001)$ (Figure 1). This correlation remained significant even after correcting for the potential confounding influence (if any) of SAPS total score $(\mathrm{p}=0.01)$. There was no significant correlation between serum HDL and the SAPS total score. None of the other lipid parameters had any significant correlation with the SAPS/SANS total scores.

\section{Discussion}

To the best of our knowledge, this is the first study to demonstrate significant negative correlation between serum HDL and a negative syndrome score in antipsychotic-naive patients with schizophrenia. Both frontal and limbic deficits underlie the negative syndrome in schizophrenia (9-11). Interestingly, HDL can potentially influence both of these regions of the brain $(5,7)$. Thus, our study observations are consistent with current theories on genesis of symptoms in patients with schizophrenia.

ApoL belongs to the HDL family that plays an important role in cholesterol transport (6). The cholesterol content of membranes is critical as it influences cellular processes by modulating gene transcription and signal transduction, both in the adult brain and during neurodevelopment (18). It is important to note that robust up-regulation of the APOL-I gene has been demonstrated in the prefrontal cortex of patients with schizophrenia (5). Interestingly, the $A P O L$ genes are clustered on chromosome 22q12 (5). Several reports have shown this chromosomal region to be linked with schizophrenia $(19,20)$. Another factor that makes this chromosomal region critical is the fact that the velocardiofacial syndrome (VCFS) has been linked to a region close by on chromosome 22q11 $(21,22)$. A significant proportion of patients with VCFS experience schizophrenia-like psychoses $(21,22)$. All these observations further strengthen the possible link between serum HDL and the pathogenesis of schizophrenia.

Some of the methodological strengths of this study include examination of antipsychotic-naive patients with schizophrenia, rigorous procedures to establish the diagnosis, good inter-rater reliability for assessment of symptoms, raters being blinded to the study hypothesis and lipid parameters, and stringent evaluation of patients to avoid potential confounding effects of other medical diseases on lipids. Some of the potential study limitations include i) lack of concurrent evaluation of brain morphometry in these patients which might have ascertained further the influence of serum HDL on negative syndrome, ii) assessment of the apoL moiety of lipoproteins would have further elucidated the pathogenetic mechanisms, and iii) lack of comprehensive evaluation of the dietary pattern in patients with schizophrenia.

In summary, the results of this study suggest a significant inverse relationship between serum HDL and the negative syndrome in schizophrenia. Further studies are needed to extend this finding by concurrent assessment of ApoL parameters as well as brain structure/function. 


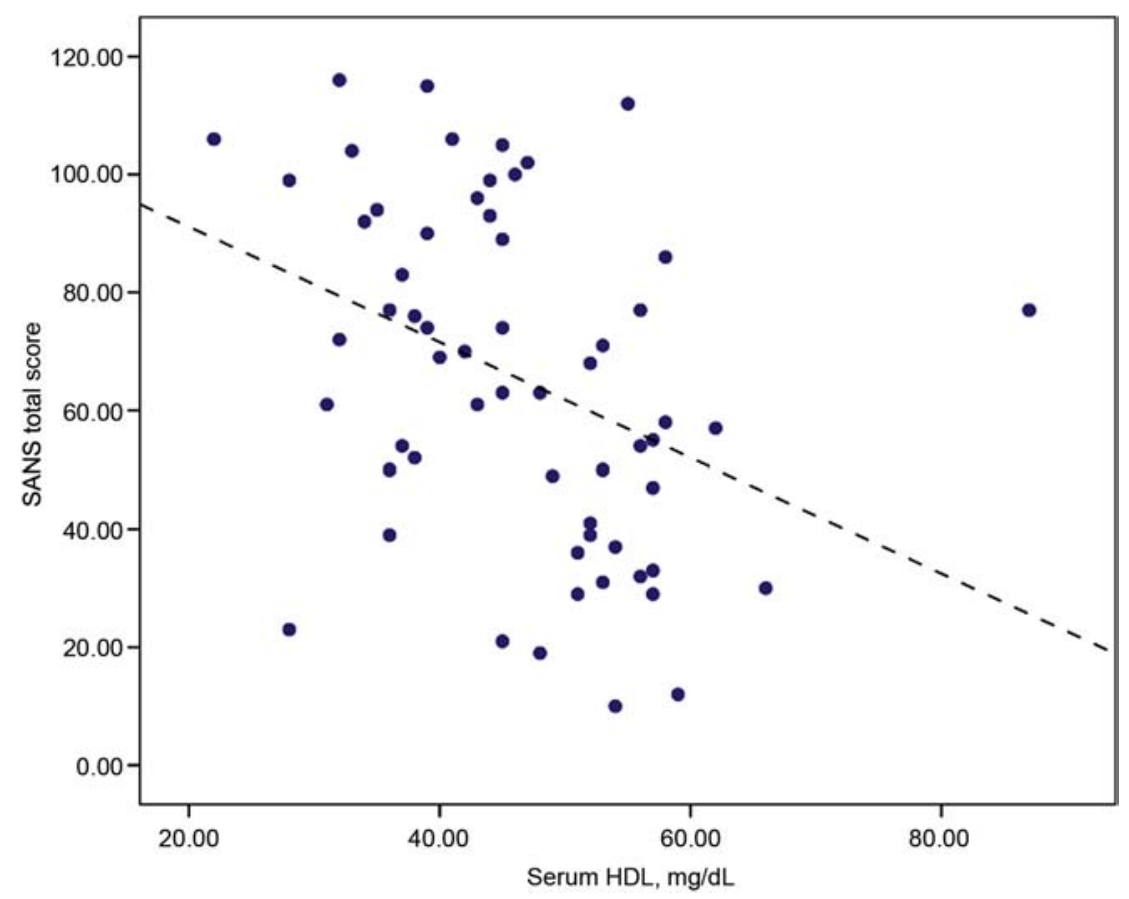

Figure 1 Significant negative correlation between serum HDL and SANS total score in antipsychotic-naive patients with schizophrenia $(\mathrm{n}=60)$.

To convert conventional HDL values to SI units, multiply with 0.02586 .

\section{Conflict of interest statement}

Authors' conflict of interest disclosure: The authors stated that there are no conflicts of interest regarding the publication of this article.

Research funding: RA was supported by the Indian Council of Medical Research and SK was supported by the Department of Biotechnology (India) - Innovative Young Biotechnologist Award Scheme through research grants [ICMR-GV-039 \& DBT-GV-040, respectively] awarded to GV.

Employment or leadership: None declared.

Honorarium: None declared.

\section{References}

1. Berger GE, Wood SJ, Pantelis C, Velakoulis D, Wellard RM, McGorry PD. Implications of lipid biology for the pathogenesis of schizophrenia. Aust N Z J Psychiatry 2002;36:355-66.

2. Horrobin DF. Lipid metabolism, human evolution and schizophrenia. Prostaglandins Leukot Essent Fatty Acids 1999;60: 431-7.

3. Sutcliffe JG, Thomas EA. The neurobiology of apolipoproteins in psychiatric disorders. Mol Neurobiol 2002;26:369-88.

4. Duchateau PN, Pullinger CR, Cho MH, Eng C, Kane JP. Apolipoprotein L gene family: tissue-specific expression, splicing, promoter regions; discovery of a new gene. J Lipid Res 2001; 42:620-30.

5. Mimmack ML, Ryan M, Baba H, Navarro-Ruiz J, Iritani S, Faull $\mathrm{RL}$, et al. Gene expression analysis in schizophrenia: reproducible up-regulation of several members of the apolipoprotein $\mathrm{L}$ family located in a high-susceptibility locus for schizophrenia on chromosome 22. Proc Natl Acad Sci USA 2002;99:4680-5.
6. Duchateau PN, Pullinger CR, Orellana RE, Kunitake ST, NayaVigne J, O'Connor PM, et al. Apolipoprotein L, a new human high density lipoprotein apolipoprotein expressed by the pancreas. Identification, cloning, characterization, and plasma distribution of apolipoprotein L. J Biol Chem 1997;272:2557682.

7. Wolf H, Hensel A, Arendt T, Kivipelto M, Winblad B, Gertz HJ. Serum lipids and hippocampal volume: the link to Alzheimer's disease? Ann Neurol 2004;56:745-8.

8. Corrigan FM, Mowat B, Skinner ER, Van Rhijn AG, Cousland G. High density lipoprotein fatty acids in dementia. Prostaglandins Leukot Essent Fatty Acids 1998;58:125-7.

9. Venkatasubramanian G, Jayakumar PN, Gangadhar BN, Keshavan MS. Automated MRI parcellation study of regional volume and thickness of prefrontal cortex (PFC) in antipsychotic-naive schizophrenia. Acta Psychiatr Scand 2008;117:42031.

10. Harrison PJ. The hippocampus in schizophrenia: a review of the neuropathological evidence and its pathophysiological implications. Psychopharmacology (Berl) 2004;174:151-62.

11. Weiner I. The "two-headed" latent inhibition model of schizophrenia: modeling positive and negative symptoms and their treatment. Psychopharmacology (Berl) 2003;169:257-97.

12. Sheehan DV, Lecrubier Y, Sheehan KH, Amorim P, Janavs J, Weiller E, et al. The Mini-International Neuropsychiatric Interview (M.I.N.I.): the development and validation of a structured diagnostic psychiatric interview for DSM-IV and ICD-10. J Clin Psychiatry 1998;59(Suppl 20):22-33.

13. Andreasen NC. The Scale for the Assessment of Positive Symptoms (SAPS). Iowa City, IA: University of Iowa; 1984.

14. Andreasen NC. The Scale for the Assessment of Negative Symptoms (SANS). Iowa City, IA: University of Iowa; 1983.

15. Mohan V, Deepa R, Deepa M, Somannavar S, Datta M. A 
simplified Indian Diabetes Risk Score for screening for undiagnosed diabetic subjects. J Assoc Physicians India 2005;53: 759-63.

16. Venkatasubramanian G, Chittiprol S, Neelakantachar N, Naveen MN, Thirthall J, Gangadhar BN, et al. Insulin and insulin-like growth factor-1 abnormalities in antipsychotic-naive schizophrenia. Am J Psychiatry 2007;164:1557-60.

17. Guy W. Abnormal Involuntary Movements Scale (AIMS). ECDEU Assessment Manual for Pharmacology: US Dept of Health, Education, and Welfare, Rockville, MD; 1976.

18. Dietschy JM, Turley SD. Cholesterol metabolism in the brain. Curr Opin Lipidol 2001;12:105-12.

19. Schwab SG, Lerer B, Albus M, Maier W, Hallmayer J, Fimmers
$\mathrm{R}$, et al. Potential linkage for schizophrenia on chromosome 22q12-q13: a replication study. Am J Med Genet 1995;60: $436-43$.

20. Takahashi S, Ohtsuki T, Yu SY, Tanabe E, Yara K, Kamioka $\mathrm{M}$, et al. Significant linkage to chromosome $22 \mathrm{q}$ for exploratory eye movement dysfunction in schizophrenia. Am J Med Genet B Neuropsychiatr Genet 2003;123B:27-32.

21. Gothelf D. Velocardiofacial syndrome. Child Adolesc Psychiatr Clin N Am 2007;16:677-93.

22. Feinstein C, Eliez S, Blasey C, Reiss AL. Psychiatric disorders and behavioral problems in children with velocardiofacial syndrome: usefulness as phenotypic indicators of schizophrenia risk. Biol Psychiatry 2002;51:312-8. 